\title{
Applications of MALDI-TOF Mass Spectrometry for the Routine Identification of Microorganisms \\ Rodríguez-Sánchez Belén ${ }^{1,2,3}$ \\ ${ }^{1}$ Clinical Microbiology and Infectious Diseases Department, Hospital General Universitario Gregorio Marañón, Spain \\ ${ }^{2}$ Instituto de Investigación Sanitaria Gregorio Marañón, Spain \\ ${ }^{3}$ CIBER de Enfermedades Respiratorias, Madrid, Spain
}

\begin{abstract}
The emerging of MALDI-TOF Mass Spectrometry as a diagnostic tool for the identification of micro-organism has transformed most microbiology laboratories, where phenotypic and cumbersome conventional techniques are still applied currently. Its implementation has allowed high-throughput, rapid, inexpensive and accurate identification of the most clinically relevant bacteria, yeast and mold species [1-5].

The last decade has witnessed how MALDI-TOF MS was increasingly applied for a higher number of microorganisms, available databases grew and sample preparations procedures improved allowing not just the identification of the organism, but also their antibiotic or antifungal susceptibility pattern [6-10] until, recently, several studies have demonstrated MALDI-TOF usefulness for epidemiological studies $[11,12]$.
\end{abstract}

\section{Publication History:}

Received: December 16, 2017

Accepted: February 08, 2018

Published: February 10, 2018

\section{Keywords:}

Poliomyelitis, Virus, Vaccine, Infection, Transmission, Prevention, Public health

\section{Identification of Bacteria}

Identification of bacterial isolates using MALDI-TOF MS has become quicker and more accurate as expertise was accumulated, sample proceedings improved and commercial databases updated with more reference protein spectra, allowing a better discrimination from closely-related species. Direct identification of commonly encountered bacteria could be performed from single colonies grown on different agar plates with highly accurate results $[1,13]$. Besides, the implementation of the on-plate protein extraction step allowed the identification of other groups of microorganisms that pose difficulties for a reliable identification, such as uncommon species or those with special growth requirements [3].

The little amount of bacterial biomass required for MALDI-TOF MS identification is suitable for the rapid identification of those micro-organisms displaying low-growth rate such as the anaerobic bacteria. In this field, great improvements have developed in the last few years. A multicenter study has been published recently showing a high correlation between MALDI-TOF MS and DNA sequencing identification [5]. Besides, the availability of a high number of unusual anaerobic isolates by the researchers within the multicenter project also allowed the improvement of the anaerobic database provided by Bruker Daltonics (Bremen, Germany). Other authors have also reported rapid, accurate and inexpensive identification of a wide variety of anaerobic isolates directly from single colonies and with no need to perform protein extraction $[14,16]$. This method yields a final identification within minutes and avoids further molecular analysis and can also be performed directly from clinical samples, such as blood cultures [16].

In this regard, several studies have demonstrated that MALDITOF MS can be easily and efficiently applied for the identification of microorganisms from blood cultures. Applying a standardized and easy-to-perform centrifuge and wash protocol, microorganisms can be obtained from both types of samples and either directly identified with MALDI-TOF MS by on-plate extraction with formic acid $[17,18]$ or by using a commercial lysis buffer (Sepsityper, Bruker Daltonics, Bremen, Germany) and a standard protein extraction step with formic-acid/acetonitrile [19]. Both approaches have rendered between $81.4-87.0 \%$ blood culture bottles correctly identified at the species level when the score values recommended by the manufacturer are applied. However, the rate of correct identifications increases up to $95 \%$ when the cut-off for species-level identification is lowered to 1.8 , with no misidentifications detected.

Despite the accuracy, robustness and reduced turnaround time of MALDI-TOF MS, several drawbacks have been described: (1) the capacity of MALDI-TOF MS to detect more polymicrobial infections is limited; (2) yeasts represent a challenge to MALDI-TOF MS when present in blood cultures. In the first case, MALDI-TOF MS has been reported to identify up to two different micro-organisms present in the same sample [18] but it frequently misses the species in lower concentration or fails to identify some of the microorganisms when more than two are present. In the cases when two or more species are expected according to the results of the gram staining, the final identification of the micro-organisms present in a blood culture bottle is delayed 18-24 hours until MALDI-TOF MS can be performed from the colonies grown on agar plates.

When yeasts are suspected in a blood culture sample the implementation of the sample procedure described above yields a very low rate of successful identifications. Thus, either the use of the commercial lysis buffer (Sepsityper, Bruker Daltonics) or detergents such as SDS [20] are recommended for a more effective lysis and identification of these entities.

In the case of urine samples, several studies have been published reporting the efficiency of MALDI-TOF MS for the rapid and accurate identification of microorganisms [21-23]. However, this

*Corresponding Author: Dr. Belén Rodríguez-Sánchez, Servicio de MicrobiologíaEnfermedades Infecciosas, Hospital General Universitario Gregorio Marañón, Madrid, Spain; E-mail: mbelen.rodriguez@iisgm.com

Citation: Rodríguez-Sánchez B (2018) Applications of MALDI-TOF Mass Spectrometry for the Routine Identification of Microorganisms. Int J Clin Med Microbiol 3: 129. doi: https://doi.org/10.15344/2456-4028/2018/129

Copyright: (C) 2018 Rodríguez-Sánchez. This is an open-access article distributed under the terms of the Creative Commons Attribution License, which permits unrestricted use, distribution, and reproduction in any medium, provided the original author and source are credited. 
type of sample presents sensitivity issues due to the limited number of bacteria present in a large volume and the already mentioned problems with polymicrobial samples. The hand-on time required for the processing of this kind of sample is usually too long. Thus, the benefit of its implementation is scarce when compared with performing MALDI-TOF MS from single colonies grown on agar plates after 12-hours incubation.

\section{Identification of Mycobacteria Isolates}

For a long time the genus Mycobacteria has represented a challenge for MALDI-TOF MS due to the characteristics of their cell wall and the difficulties it pose for an efficient protein extraction. Therefore, successful identification was only achieved when in-house libraries enriched with different Mycobacteria species were used [24].

However, two events rendered MALDI-TOF MS a robust and accurate tool for the identification of Mycobacterium spp.: the use of mechanical disruption of the cell wall with glass beads followed by a standard protein extraction step and the improvement of the available database [25]. Both factors have allowed the routine identification of Mycobacterial isolates, especially those species grouped as NonTuberculous Mycobacteria (NTM) since discrimination within the Mycobacterium tuberculosis complex has not been achieved yet.

Currently, the identification of Mycobacterial isolates using MALDI-TOF is widely spread, with a good number of research groups publishing high rates of correct species assignment for an increasing number of NTM species [26-28]. A recent multicenter study has demonstrated the high rate of accuracy displayed by MALDI-TOF MS, with close to $100 \%$ successful identification at the species level [29]. Besides, the implementation of sonication increased the score values obtained and allowed a reliable identification of NTM isolates. The high correlation of MALDI-TOF MS identification with reference molecular methods, robustness, accuracy and its low price per sample make this technology highly suitable for routine identification of NTM isolates. Its identification requires little hands-on time and allows a rapid and reliable identification of the isolates. This is a key factor for the early onset of the correct antibiotic therapy when required, improving the management of infected patients.

\section{Identification of Yeasts and Filamentous Fungi}

As explained above for the Mycobacterium genus, yeasts and filamentous fungi have also represented a challenging taxonomic group for MALDI-TOF MS. After years of experience with this technology and with the available updated databases, most yeasts are currently represented, even Candida auris [30]. Nowadays, most commonly encountered yeast species are easily identified with MALDI-TOF MS, even directly from blood cultures, as mentioned before (20) and they are routinely identified using this methodology [31,32].

On the other hand, filamentous fungi have been identified with MALDI-TOF MS after a long and difficult process of sample preparation improvement and in-house database building. This explains why still very few laboratories have applied this technology for the identification of filamentous fungi $[31,33,34]$.

However, the improvement of sample preparation and the good results obtained have encouraged different research groups to build their own in-house databases with well-characterized, local isolates or even to share them [35] so that identification of filamentous fungi develop an easier task to be carried out with MALDI-TOF MS and, even, to implement this methodology in the routine of the clinical microbiology laboratory $[36,37]$.

\section{Susceptibility Testing}

The huge potential of protein spectra analysis by MALDI-TOF MS has, so far, only started to show. Besides identification of microorganisms, providing reliable information about their susceptibility profile could shorten the time until a directed antibiotic/antifungal treatment is implemented. This fact has often been correlated with a better outcome of the patient and reduced hospital costs [38].

Several authors have reported promising results on this regard. A methodological paper was published in 2012 showing how susceptibility to different beta lactam antibiotics [6]. The protocol consists of incubating the bacterial pellet in the present of the antibiotic tested and a peak shift is seen due to the breakage of the beta lactam ring when beta-lactamases are present. This study has represented a framework for further development and implementation of susceptibility testing using MALDI-TOF MS. Together with the identification of microorganisms, combination of both tests carried out with the same instrument- have shown to provide useful information in a fast, inexpensive and reliable manner. Estimated turnaround time for identification and susceptibility profile is $30-45 \mathrm{~min}$.

The development of this methodology has allowed different authors the identification of resistance mechanisms from bacteria present in blood culture $[39,40]$. Identification of the microorganisms present in blood samples and susceptibility testing has been demonstrated to be feasible with MALDI-TOF MS and a high correlation with other reference techniques has been shown.

The emerging of carbapenem-resistant isolates has also become a global health problem. The implementation of MALDI-TOF MS for the detection of this type of resistance mechanisms has also been shown for different bacterial genera, with promising results [4042]. Recently, a commercial kit has also been commercialized for the easy and standardized detection of imipenem-resistant strains (MBT STAR-Carba IVD Kit -STAR - Selective Testing of Antibiotic Resistance, Bruker Daltonics, Bremen, Germany).

Finally, antifungal susceptibility testing has also been performed with MALDI-TOF MS [43-45]. Different resistance mechanisms have been assayed and their results demonstrate once again the capacity of MALDI-TOF MS to detect them in a quick, efficient and inexpensive manner.

\section{Conclusions}

MALDI-TOF MS technology has emerged as a rapid, reliable and robust tool for the identification of microorganisms. Its user-friendly technology that requires very little training hands-on time to be skillfully performed has rendered it as a must-have instrument in most clinical microbiology laboratories. Once users have started to accumulate expertise on its application to different bacterial species and sample types, it has been implemented for the identification of a wide range of bacteria, mycobacteria, yeasts or molds. Besides, other applications such as susceptibility testing or typing have also been feasible with the same technology. This fact has shown the great 
Citation: Rodríguez-Sánchez B (2018) Applications of MALDI-TOF Mass Spectrometry for the Routine Identification of Microorganisms. Int J Clin Med Microbiol 3: 129. doi: https://doi.org/10.15344/2456-4028/2018/129

Page 3 of 4

flexibility of MALDI-TOF MS and its potential for future applications. The revolution we have witness in the last decade will probably keep on going for a little while as MALDI-TOF MS unfolds its endless purposes.

\section{Competing Interests}

The authors declares that they have no competing interests.

\section{References}

1. Seng P, Drancourt M, Gouriet F, La Scola B, Fournier PE, et al. (2009) Ongoing revolution in bacteriology: routine identification of bacteria by matrix assisted laser desorption ionization time-of-flight mass spectrometry. Clin Infect Dis 49: 543-551.

2. Becker PT, de Bel A, Martiny D, Ranque S, Piarroux R, et al. (2014) Identification of filamentous fungi isolates by MALDI-TOF mass spectrometry: clinical evaluation of an extended reference spectra library. Med Mycol 52: 826834

3. Rodríguez-Sánchez $B$, Marín M, Sánchez-Carrillo C, Cercenado E, Ruiz A, et al. (2014) Improvement of matrix-assisted laser desorption/ionization time-of-flight mass spectrometry identification of difficult-to-identify bacteria and its impact in the workflow of a clinical microbiology laboratory DiagnMicrobiol Infect Dis 79: 1-6.

4. Turhan O, Ozhak-Baysan B, Zaragoza O, Er H, Sarıtas ZE, et al. (2017) Evaluation of MALDI-TOF-MS for the Identification of Yeast Isolates Causing Bloodstream Infection. Clin Lab 63: 699-703.

5. Veloo ACM, Jean-Pierre H, Justesen US, Morris T, Urban E, et al. (2017) A multi-center ring trial for the identification of anaerobic bacteria using MALDI-TOF MS. Anaerobe 48: 94-97.

6. Sparbier K, Schubert S, Weller U, Boogen C, Kostrzewa M, et al. (2012) Matrixassisted laser desorption ionization-time of flight mass spectrometrybased functional assay for rapid detection of resistance against $\beta$-lactam antibiotics. J ClinMicrobiol 50: 927-937.

7. Hrabák J (2015) Detection of carbapenemases using matrix-assisted laser desorption/ionization time-of-flight mass spectrometry (MALDI-TOF MS) meropenem hydrolysis assay. Methods Mol Biol 1237: 91-96.

8. Jung JS, Hamacher C, Gross B, Sparbier K, Lange C, et al. (2016) Evaluation of a Semiquantitative Matrix-Assisted Laser Desorption Ionization-Time of Flight Mass Spectrometry Method for Rapid Antimicrobial Susceptibility Testing of Positive Blood Cultures. J ClinMicrobiol 54: 2820-2824.

9. Ceyssens PJ, Soetaert K, Timke M, Bossche AV, Sparbier K, et al. (2017) Matrix-Assisted Laser Desorption Ionization-Time of Flight Mass Spectrometry for Combined Species Identification and Drug Sensitivity Testing in Mycobacteria. J ClinMicrobiol 55: 624-634.

10. Oviaño M, Ramírez CL, Barbeyto LP, Bou G (2017) Rapid direct detection of carbapenemase-producing Enterobacteriaceae in clinical urine samples by MALDI-TOF MS analysis. J AntimicrobChemother 72: 1350-1354.

11. Egli A, Tschudin-Sutter S, Oberle M, Goldenberger D, Frei R, et al. (2015) Matrix-assisted laser desorption/ionization time of flight mass-spectrometry (MALDI-TOF MS) based typing of extended-spectrum $\beta$-lactamase producing E. coli--a novel tool for real-time outbreak investigation.PLoS One 10: e0120624.

12. Oberle M, Wohlwend N, Jonas D, Maurer FP, Jost G, et al. (2016) The Technical and Biological Reproducibility of Matrix-Assisted Lase Desorption Ionization-Time of Flight Mass Spectrometry (MALDI-TOF MS) Based Typing: Employment of Bioinformatics in a Multicenter Study. PLoS One 11: e0164260.

13. Bizzini A, Durussel C, Bille J, Greub G, Prod'hom G, et al. (2010) Performance of matrix-assisted laser desorption ionization-time of flight mass spectrometry for identification of bacterial strains routinely isolated in a clinical microbiology laboratory. J ClinMicrobiol 48: 1549-1554.

14. Rodríguez-Sánchez B, Alcalá L, Marín M, Ruiz A, Alonso E, et al. (2016) Evaluation of MALDI-TOF MS (Matrix-Assisted Laser Desorption-Ionization Time-of-Flight Mass Spectrometry) for routine identification of anaerobic bacteria. Anaerobe 42: 101-107.
15. Veloo AC, de Vries ED, Jean-Pierre H, Justesen US, Morris T, et al. (2016) The optimization and validation of the Biotyper MALDI-TOF MS database for the identification of Gram-positive anaerobic cocci. ClinMicrobiol Infect 22: 793-798.

16. Intra J, Sala MR, Falbo R, Cappellini F, Brambilla P, et al. (2016) Reducing time to identification of aerobic bacteria and fastidious micro-organisms in positive blood cultures. Lett ApplMicrobiol 63: 400-405.

17. Christner M, Rohde H, Wolters M, Sobottka I, Wegscheider K, et al. (2010) Rapid identification of bacteria from positive bloodculture bottles by use of matrix-assisted laser desorption-ionization time of flight mass spectrometry fingerprinting. J ClinMicrobiol 48: 1584-1591.

18. Rodríguez-Sánchez B, Sánchez-Carrillo C, Ruiz A, Marín M, Cercenado E, et al. (2014) Direct identification of pathogens from positive blood cultures using matrix-assisted laser desorption-ionization time-of-flight mass spectrometry. ClinMicrobiol Infect 20: O421-0427.

19. Lagacé-Wiens PR, Adam HJ, Karlowsky JA, Nichol KA, Pang PF, et al. (2012) Identification of blood culture isolates directly from positive blood cultures by use of matrix-assisted laser desorption ionization-time of flight mass spectrometry and a commercial extraction system: analysis of performance, cost, and turnaround time. J ClinMicrobiol 50: 3324-3328.

20. Bidart M, Bonnet I, Hennebique A, Kherraf ZE, Pelloux $H$, et al. (2015) An in-house assay is superior to Sepsityper for direct matrix-assisted laser desorption ionization-time of flight (MALDI-TOF) mass spectrometry identification of yeast species in blood cultures. J ClinMicrobiol 53: 17611764

21. Zboromyrska Y, Rubio E, Alejo I, Vergara A, Mons A, et al. (2016) Development of a new protocol for rapid bacterial identification and susceptibility testing directly from urine samples. Clin Microbiol Infect 22: 561.

22. Veron L, Mailler S, Girard V, Muller BH, L'Hostis G, et al. (2015) Rapid urine preparation prior to identification of uropathogens by MALDI-TOF MS. Eur J ClinMicrobiol Infect Dis 34: 1787-1795.

23. Íñigo M, Coello A, Fernández-Rivas G, Rivaya B, Hidalgo J, et al. (2016) Direct Identification of Urinary Tract Pathogens from Urine Samples, Combining Urine Screening Methods and Matrix-Assisted Laser Desorption IonizationTime of Flight Mass Spectrometry. J ClinMicrobiol 54: 988-993.

24. El Khéchine A, Couderc C, Flaudrops C, Raoult D, Drancourt $M$, et al. (2011) Matrix-assisted laser desorption/ionization time-of-flight mass spectrometry identification of mycobacteria in routine clinical practice. PLoS One 6: e24720.

25. Rodríguez-Sánchez $B$, Ruiz-Serrano MJ, Ruiz A, Timke $M$, Kostrzewa $M$ et al. (2016) Evaluation of MALDI Biotyper Mycobacteria Library v3.0 fo Identification of Nontuberculous Mycobacteria. J ClinMicrobiol 54: 11441147.

26. Pranada AB, Witt E, Bienia M, Kostrzewa M, Timke $M$, et al. (2017) Accurate differentiation of Mycobacterium chimaera from Mycobacterium intracellulare by MALDI-TOF MS analysis. J Med Microbiol 66: 670-677

27. Alcaide F, Peña MJ, Pérez-Risco D, Camprubi D, Gonzalez-Luquero L, et al. (2017) Increasing isolation of rapidly growing mycobacteria in a low-incidence setting of environmental mycobacteria, 1994-2015.Eur J ClinMicrobiol Infect Dis 36: 1425-1432.

28. Fangous MS, Mougari F, Gouriou S, Calvez E, Raskine L, Cambau E, et al. (2014) Classification algorithm for subspecies identification within the Mycobacterium abscessus species, based on matrix-assisted laser desorption ionization-time of flight mass spectrometry. J ClinMicrobiol 52 : 3362-3369.

29. Alcaide F, Amlerová J, Bou G, Ceyssens PJ, Coll P, et al. (2017) How To: Identify Non-Tuberculous Mycobacterium Species By Using Maldi-Tof Mass Spectrometry. ClinMicrobiol Infect pii: S1198-743X(17)30643-2.

30. Kathuria S, Singh PK, Sharma C, Prakash A, Masih A et al. (2015) Multidrug-Resistant Candida auris Misidentified as Candida haemulonii: Characterization by Matrix-Assisted Laser Desorption Ionization-Time of Flight Mass Spectrometry and DNA Sequencing and Its Antifungal Susceptibility Profile Variability by Vitek 2, CLSI Broth Microdilution, and Etest Method. J ClinMicrobiol 53:1823-1830.

31. Posteraro B, De Carolis E, Vella A, Sanguinetti M (2013) MALDI-TOF mass spectrometry in the clinical mycology laboratory: identification of fungi and beyond. Expert Rev Proteomics 10: 151-164.

32. Wang W, Xi H, Huang M, Wang J, Fan M, et al. (2014) Performance of mass spectrometric identification of bacteria and yeasts routinely isolated in a clinical microbiology laboratory using MALDI-TOF MS. J Thorac Dis 6: 524533. 
Citation: Rodríguez-Sánchez B (2018) Applications of MALDI-TOF Mass Spectrometry for the Routine Identification of Microorganisms. Int J Clin Med Microbiol 3: 129. doi: https://doi.org/10.15344/2456-4028/2018/129

Page 4 of 4

33. Ranque $\mathrm{S}$, Normand AC, Cassagne C, Murat JB, Bourgeois N, et al. (2014) MALDI-TOF mass spectrometry identification of filamentous fungi in the clinical laboratory. Mycoses 57: 135-140.

34. De Carolis E, Posteraro B, Lass-Flörl C, Vella A, Florio AR, et al. (2012) Species identification of Aspergillus, Fusarium and Mucorales with direct surface analysis by matrix-assisted laser desorption ionization time-of-flight mass spectrometry. ClinMicrobiol Infect 18: 475-484.

35. Lau AF, Drake SK, Calhoun LB, Henderson CM, Zelazny AM, et al. (2013) Development of a clinically comprehensive database and a simple procedure for identification of molds from solid media by matrix-assisted laser desorption ionization-time of flight mass spectrometry. J ClinMicrobiol 51: 828-834.

36. Del Chierico F, Masotti A, Onori M, Fiscarelli E, Mancinelli L, et al. (2012) MALDI-TOF MS proteomic phenotyping of filamentous and other fungi from clinical origin. J Proteomics 75: 3314-3330.

37. Zvezdanova ME, Escribano $P$, Ruiz A, Martínez-Jiménez MC, Peláez T, et al (2017) Increased Species-Assignment of Filamentous Fungi using MALDITOF MS coupled with a Simplified Sample Processing and an In-House Library. Med Mycol.

38. French K, Evans J, Tanner H, Gossain S, Hussain A, et al. (2016) The Clinical Impact of Rapid, Direct MALDI-ToF Identification of Bacteria from Positive Blood Cultures. PLoS One 11: e0169332.

39. Oviaño M, Fernández B, Fernández A, Barba MJ, Mouriño C, et al. (2014) Rapid detection of enterobacteriaceae producing extended spectrum betalactamases directly from positive blood cultures by matrix-assisted laser desorption ionization-time of flight mass spectrometry. ClinMicrobiol Infect 20: $1146-1157$

40. Jung JS, Popp C, Sparbier K, Lange C, Kostrzewa M, et al. (2014) Evaluation of matrix-assisted laser desorption ionization-time of flight mass spectrometry for rapid detection of $\square$-lactam resistance in Enterobacteriaceae derived from blood cultures. J ClinMicrobiol 52: 924-930.

41. Johansson $\AA$, Nagy E, Sóki J (2014) Instant screening and verification of carbapenemase activity in Bacteroides fragilis in positive blood culture, using matrix-assisted laser desorption ionization--time of flight mass spectrometry.J Med Microbiol 63: 1105-1110.

42. Papagiannitsis $C C$, Študentová $V$, Izdebski $R$, Oikonomou O, Pfeifer $Y$, et al. (2015) Matrix-assisted laser desorption ionization-time of flight mass spectrometry meropenem hydrolysis assay with $\mathrm{NH} 4 \mathrm{HCO} 3$, a reliable tool for direct detection of carbapenemase activity. J Clin Microbiol 53: 17311735.

43. Vella A, De Carolis E, Vaccaro L, Posteraro P, Perlin DS, et al. (2013) Rapid antifungal susceptibility testing by matrix-assisted laser desorption ionization-time of flight mass spectrometry analysis. J ClinMicrobiol 51: 2964-2969.

44. De Carolis E, Vella A, Florio AR, Posteraro P, Perlin DS, et al. (2012) Use of matrix-assisted laser desorption ionization-time of flight mass spectrometry for caspofungin susceptibility testing of Candida and Aspergillus species. J ClinMicrobiol 50: 2479-2483.

45. Paul S, Singh P, A S S, Rudramurthy SM, Chakrabarti A, et al. (2017) Rapid detection of fluconazole resistance in Candida tropicalis by MALDI-TOF MS.Med Mycol. 